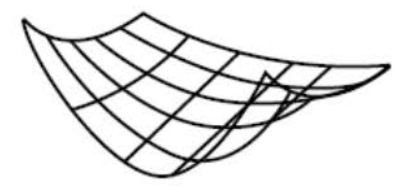

QUANTITATIVE FINANCE

RESEARCH CENTRE

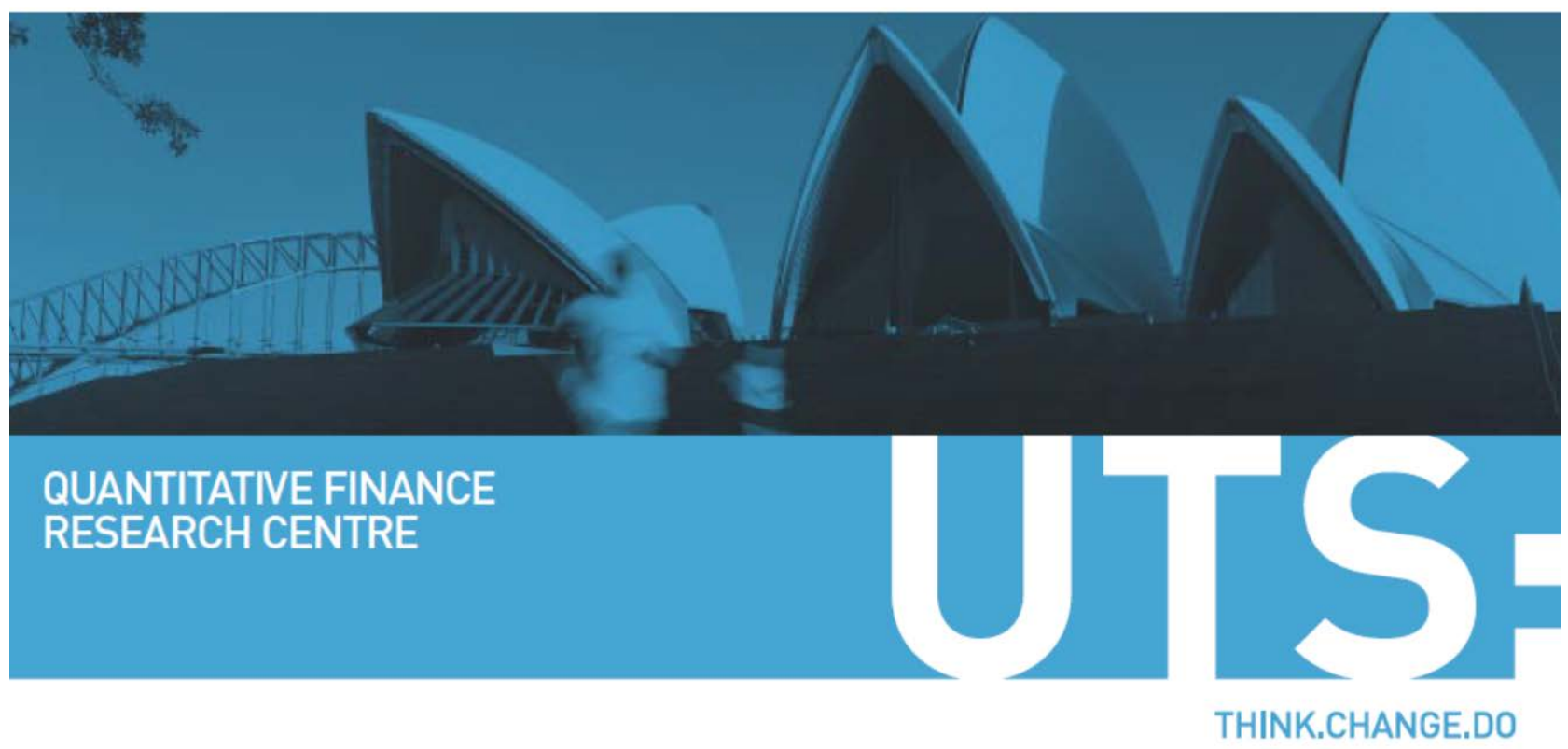

Benchmarked Risk Minimizing Hedging Strategies for Life Insurance Policies

Jin Sun and Eckhard Platen 


\title{
Benchmarked Risk Minimizing Hedging Strategies for Life Insurance Policies
}

\author{
Jin Sun ${ }^{\mathrm{a}, \mathrm{c}, *}$, Eckhard Platen ${ }^{\mathrm{a}, \mathrm{b}}$ \\ ${ }^{a}$ Faculty of Sciences, University of Technology Sydney \\ ${ }^{b}$ UTS Business School, University of Technology Sydney \\ ${ }^{c}$ Data 61, CSIRO Docklands
}

\begin{abstract}
Traditional life insurance policies offer no equity investment opportunities for the premium paid, and suffer from low returns over the long insurance terms. Modern equity-linked insurance policies offer equity investment opportunities exposed to equity market risk. To combine the low-risk of traditional policies with the high returns offered by equity-linked policies, we consider insurance policies under the benchmark approach (BA), where the policyholders' funds are invested in the growth-optimal portfolio and the locally risk-free savings account. Under the BA, life insurance policies can be delivered at their minimal costs, lower than the classical actuarial theory predicts. Due to unhedgeable mortality risk, life insurance policies cannot be fully hedged. In this case benchmarked risk-minimization can be applied to obtain hedging strategies with minimally fluctuating profit and loss processes, where the fluctuations can further be reduced through diversification.
\end{abstract}

Keywords: benchmark approach; benchmarked risk minimization; life insurance; mortality model

JEL classification: G13, G22

\section{Introduction}

Standard life insurance policies, such as term insurance policies, which pay a lump sum benefit upon death of the policyholder during a specified term; and pure endowment policies, which pay a lump sum upon survival of the policyholder at the end of a specified term; or the combination of the two, are traditionally priced under the actuarial principle of equivalence. That is, the benefits paid and the premiums received by the insurer are matched on average, after discounting by the (locally) risk-free savings account. See, e.g., Dickson et al. (2013), for a comprehensive introduction of classical actuarial pricing theory for life contingencies.

\footnotetext{
${ }^{*}$ Corresponding author

Email addresses: jin.sun@uts.edu.au (Jin Sun), eckhard.platen@uts.edu.au (Eckhard Platen)
} 
As policyholders become more interested in investment returns on their premiums, combined insurance and savings plans are becoming more popular, such as equity-linked insurance policies. These policies carry both mortality and market risks due to the explicit dependence of the benefit on some specified equity index. To alleviate market risk exposures, guaranteed benefits are often added to these contracts, such as guaranteed minimal maturity benefit (GMMB), guaranteed minimal death benefit (GMDB), etc., and combinations of these guarantees. See, e.g., Bauer et al. (2008) for a overview of such guarantee products. The addition of these guaranteed benefits partly removes the downside market risk at the expense of additional costs to the policyholder. The additional guaranteed benefits can be viewed as embedded options. The market risk, including any additional guaranteed benefits, are usually priced under an equivalent risk-neutral probability measure following the classical risk-neutral pricing theory. On the other hand, mortality risk is often considered diversifiable and priced under the real-world probability measure, following the actuarial principle of equivalence. In the pricing of insurance policies involving both market and mortality risks, the two types of risks are often assumed to be independent and separately priced under their respective pricing rules. We refer to Møller (1998) for a general approach to the risk minimization of insurance policies under independent market and mortality risks in a risk-neutral pricing framework.

As interest rates have been low across developed economies for an extended period of time since the recent financial crisis, and are expected to stay low for longer, the low interest rates imply low returns on the insurance premiums for traditional life insurance policies, or equivalently, high premiums for the same level of benefit. On the other hand, equity-linked policies are either exposed to market risk or subject to additional costs of the guarantees. We demonstrate how to overcome these difficulties by following the benchmark approach (BA) proposed by Platen and Heath (2006), and consider the minimal producing costs of the life insurance policies. In particular, the standard term insurance and pure endowment policies may be produced less expensively than the classical theory implies. Additionally, the aim of benefitting from the superior expected long-term growth of the equity market is naturally implemented by the strategies we derive.

The BA assumes that in a given investment universe, there exists a unique growth-optimal portfolio (GP), which we call the benchmark. The BA takes the GP as numeraire, or benchmark, such that any benchmarked (in units of the GP denominated) nonnegative portfolio price process assumes zero expected instantaneous returns. The GP achieves the highest expected instantaneous growth rate among all nonnegative portfolios in the investment universe by maximizing the expected log-utility of terminal wealth. The GP is a well-diversified portfolio that draws on all tradable risk factors and the corresponding risk premiums to achieve the growth-optimality. Under the BA, the real-world pricing formula of any nonnegative, replicable contingent claim, which makes the price denominated in units of the GP a martingale, represents the minimal possible replication cost of the claim. 
The purpose of this paper is to propose the pricing of standard life insurance policies under the BA, where the GP of the financial market may be approximated by a well-diversified equity index. Our paper extends the approach described by Møller (1998), where the formula-

\section{The Financial Market Model}

In this section, we introduce the model for the financial market and give a brief overview of the BA. We consider a general diffusive financial market model with uncertainties driven by a d-dimensional Brownian motion $\boldsymbol{W}$, with $\boldsymbol{W}(t)=\left\{\left(W(t)^{1}, \ldots, W(t)^{d}\right)^{\top}, t \in[0, T]\right\}$, defined on a filtered probability space $(\Omega, \mathcal{F}, \mathbb{F}, \mathbb{P})$, where $T$ is some fixed time horizon, and the filtration $\mathbb{F}=\left\{\mathcal{F}_{t}, t \in[0, T]\right\}$ satisfies the usual conditions of right continuity and completeness, and models the accumulation of information over time; see Karatzas and Shreve (1991). We assume that there exists a locally risk-free savings account $S^{0}(t)$ and $m$ nonnegative risky primary security accounts $\boldsymbol{S}(t)=\left(S^{1}(t), \ldots, S^{m}(t)\right)^{\top}$ satisfying the vector stochastic differential equation $(\mathrm{SDE})$

$$
d \boldsymbol{S}(t)=\boldsymbol{S}(t)(a(t) d t+b(t) \cdot d \boldsymbol{W}(t)), \quad t \in[0, T]
$$

where $a(t)$ is the instantaneous drift vector and $b(t)$ the instantaneous volatility matrix, which both are assumed to be predictable and such that a unique strong solution of the above system of SDEs exists. We assume that all dividends and interests are reinvested. Without loss of generality, we further assume that $S^{0}(t) \equiv 1$. This means that we denominate all security prices in units of the locally risk-free savings account. In practice, the locally risk-free savings account may be approximated by the money market account that invests in short-term T-bills 
in a rolling manner. Thus in our notation, all primary security accounts are discounted by the locally risk-free savings account.

We denote by $S^{\pi}$ the value process of a strictly positive, self-financing portfolio with portfolio weights $\boldsymbol{\pi}(t)=\left(\pi^{1}(t), \ldots, \pi^{m}(t)\right)^{\top}, t \in[0, T]$, which invests at time $t$ a fraction $\pi^{j}(t)$ of the total wealth in the $j$ th primary security account, and the remaining wealth in the locally risk-free savings account. The value process satisfies then the SDE

$$
\frac{d S^{\boldsymbol{\pi}}(t)}{S^{\boldsymbol{\pi}}(t)}=\boldsymbol{\pi}(t)^{\top}(a(t) d t+b(t) \cdot d \boldsymbol{W}(t)), \quad t \in[0, T]
$$

By Ito's formula, the SDE for the log-price is of the form

$$
d \log S^{\boldsymbol{\pi}}(t)=\boldsymbol{\pi}(t)^{\top}\left(\left(a(t)-\frac{1}{2} b(t) b(t)^{\top} \boldsymbol{\pi}(t)\right) d t+b(t) \cdot d \boldsymbol{W}(t)\right), \quad t \in[0, T] .
$$

We consider the growth-optimal portfolio (GP) $S^{\pi^{*}}$ of this investment universe for which the instantaneous expected growth rate, that is, the drift of (3), is maximized for all $t$. This is achieved by setting the optimal portfolio weights $\boldsymbol{\pi}^{*}(t)$ to

$$
\boldsymbol{\pi}^{*}(t)=\underset{\boldsymbol{\pi}}{\arg \max } \boldsymbol{\pi}^{\top}\left(a(t)-\frac{1}{2} b(t) b(t)^{\top} \boldsymbol{\pi}\right), \quad t \in[0, T] .
$$

We assume that a solution to (4) exists a.s. for all $t \in[0, T]$. One potential such solution is given by

$$
\boldsymbol{\pi}^{*}(t)=\left(b(t) b(t)^{\top}\right)^{+} a(t), \quad t \in[0, T]
$$

where $\left(b(t) b(t)^{\top}\right)^{+}$denotes the Moore-Penrose generalized inverse of the self-adjoint matrix $b(t) b(t)^{\top}$. Note that the value process of the GP is unique, however, the fractions may vary due to potential redundancies in the primary security accounts

For the market model to be viable, we assume that the GP process, denoted as $S^{*}(t):=$ $S^{\pi^{*}}(t), t \in[0, T]$, with $\pi^{*}(t)$ given by (5), exists and is strictly positive. By substituting (5) into (2), we obtain the SDE

$$
\frac{d S^{*}(t)}{S^{*}(t)}=\|\boldsymbol{\theta}(t)\|^{2} d t+\boldsymbol{\theta}(t) \cdot d \boldsymbol{W}(t), \quad t \in[0, T]
$$

where $\boldsymbol{\theta}(t)=b(t)^{\top} \boldsymbol{\pi}^{*}(t)$. The above SDE can further be written as

$$
d S^{*}(t)=\alpha(t) d t+\sqrt{\alpha(t) S^{*}(t)} d W^{*}(t), \quad t \in[0, T],
$$

where the drift $\alpha(t)=\|\boldsymbol{\theta}(t)\|^{2} S^{*}(t)$ is assumed to be strictly positive, and $W^{*}(t)$, defined by 
the SDE

$$
d W^{*}(t)=\frac{\boldsymbol{\theta}(t)}{\|\boldsymbol{\theta}(t)\|} \cdot d \boldsymbol{W}(t) \quad t \in[0, T],
$$

with $W^{*}(0)=0$, forms a standard Brownian motion by Levy's characterization theorem. So far, we only re-parametrized the GP dynamics different to the common volatility modeling specification. Note that the above drift $\alpha(t)$ can be, at this stage, still very general. Later on, we will make this drift more specific, which yields then a proper model.

The GP is the unique portfolio which, when used as numeraire or benchmark, makes any benchmarked portfolio process $\hat{S}^{\pi}$, defined as $\hat{S}^{\pi}(t)=\frac{S^{\pi}(t)}{S^{*}(t)}$, a local martingale. If we assume the portfolio process to be nonnegative, the benchmarked portfolio process becomes a supermartingale by Fatou's lemma. Given an $\mathcal{F}_{T}$-measurable nonnegative contingent claim $H \geq 0$ with maturity $T$, its, so called, fair price process under the BA is given by the real-world pricing formula as

$$
V_{H}(t)=E_{t}\left(\frac{S^{*}(t)}{S^{*}(T)} H\right), \quad t \in[0, T],
$$

where $E_{t}(\cdot)=E\left(\cdot \mid \mathcal{F}_{t}\right)$ denotes the $\mathcal{F}_{t}$-conditional expectation under the real-world probability measure $\mathbb{P}$. The benchmarked fair price process, defined as $\hat{V}_{H}(t)=\frac{V_{H}(t)}{S^{*}(t)}$, forms then a nonnegative $(\mathbb{F}, \mathbb{P})$-martingale. The benchmarked fair price process $\hat{V}_{H}$, if replicable, represents the least expensive portfolio among all benchmarked nonnegative self-financing replication portfolios, which form supermartingales. For example, consider a zero-coupon bond that pays one unit of the locally risk-free savings account at maturity $T$. Following (9), the fair price process of this bond is given by

$$
P^{*}(t, T)=E_{t}\left(\frac{S^{*}(t)}{S^{*}(T)}\right), \quad t \in[0, T] .
$$

We further assume that the GP is a scalar Markov process, and $P^{*}(t, T)$ is a suitably smooth function of $S^{*}(t)$ and $t$. Hence the fair zero-coupon bond (10) can be synthesized by holding a replication portfolio of value $P^{*}(t, T)$ at $t \in[0, T]$, consisting of a number of

$$
\delta^{*}(t)=\frac{\partial P^{*}(t, T)}{\partial S^{*}(t)}
$$

units of the GP, and a number of $P^{*}(t, T)-\delta^{*}(t) S^{*}(t)$ units of the locally risk-free savings account. By applying Ito's formula to the benchmarked fair zero-coupon bond price $\hat{P}^{*}(t, T)=$ $\frac{P^{*}(t, T)}{S^{*}(t)}$, and considering $\sqrt{7}, \sqrt{11}$, as well as, the fact that $\hat{P}^{*}(t, T)$ forms a martingale with zero drift, we obtain the $\operatorname{SDE}$ for $\hat{P}^{*}(t, T)$ as

$$
d \hat{P}^{*}(t, T)=\sqrt{\frac{\alpha(t)}{S^{*}(t)}}\left(\delta^{*}(t)-\hat{P}^{*}(t, T)\right) d W^{*}(t), \quad t \in[0, T]
$$


with $\hat{P}^{*}(T, T)=S^{*}(T)^{-1}$. Note that by applying Ito's formula to $P^{*}(t, T)=S^{*}(t) \hat{P}^{*}(t, T)$, we obtain

$$
d P^{*}(t, T)=\delta^{*}(t) d S^{*}(t), \quad t \in[0, T],
$$

which verifies the hedge ratio (11) for replicating the fair zero-coupon bond.

\section{The Mortality Model}

In this section we turn our attention to the mortality model, and consider pricing of insurance policies under the combined model. By incorporating mortality risk associated with insurance policies, the financial market becomes incomplete, and insurance policies cannot be fully replicated by trading the financial securities alone.

We consider a portfolio of life insurance policies sharing the same policy details and maturity $T$, written for a homogeneous group of individual policyholders with independent and identically distributed remaining life times $T_{i}>0, i=1, \ldots, L$, where $L$ is the total number of policyholders in the portfolio. We assume that the remaining life times are independent of the financial market, and share the common survival function given in terms of the hazard rate as

$$
s(t):=\mathbb{P}\left(T_{i}>t\right)=\exp \left(-\int_{0}^{t} \mu(\tau) d \tau\right), \quad t \in[0, T],
$$

where $\mu(t) \geq 0, t \in[0, T]$ is the left-continuous force of mortality process common to all policyholders. For simplicity, we assume the hazard rate process to be deterministic. We define the conditional survival function $s(t, u)$ as

$$
s(t, u):=\mathbb{P}\left(T_{i}>u \mid T_{i}>t\right)=\exp \left(-\int_{t}^{u} \mu(\tau) d \tau\right), \quad 0 \leq t \leq u,
$$

i.e., the conditional probability that a policyholder survives through $u \geq t$, given that he or she survived through $t$. In terms of standard actuarial notation, we have $s(t, u)={ }_{u-t} p_{x+t}$ for cohorts aged $x$ at $t=0$.

The counting process $N(t), t \in[0, T]$, of the total number of deaths within the portfolio is given by

$$
N(t):=\sum_{i=1}^{L} \mathbb{1}_{\left\{T_{i} \leq t\right\}}, \quad t \in[0, T],
$$

with $N(0)=0$. Here $\mathbb{1}_{\{\cdot\}}$ denotes the indicator function for an event. Evidently, $N$ is right continuous with left-hand limits. We assume the current information $\mathcal{F}_{t}$ contains all information available at time $t$ regarding the financial market, the hazard rate and the survival of individual policyholders. Under this assumption, $N$ is adapted to $\mathbb{F}$. The stochastic 
intensity process $\lambda(t), t \in[0, T]$, of the mortality counting process $N$ is evidently given by

$$
\lambda(t):=\lim _{h \downarrow 0} \frac{1}{h} E_{t}(N(t+h)-N(t))=(L-N(t)) \mu(t), \quad t \in[0, T),
$$

and the compensated counting process

$$
M(t):=N(t)-\int_{0}^{t} \lambda\left(u_{-}\right) d u=N(t)-\int_{0}^{t}\left(L-N\left(u_{-}\right)\right) \mu(u) d u, \quad t \in[0, T]
$$

is an $(\mathbb{F}, \mathbb{P})$-martingale.

We assume that the $i$-th individual life insurance policy pays a lump-sum benefit $H_{i}$ at $T_{i} \wedge T:=\min \left(T_{i}, T\right)$, where $H_{i}$ is $\mathcal{F}_{T_{i} \wedge T}$-measurable, and does not depend on other policyholders' survival information. Examples of such policies include standard term insurance, pure endowment, endowment insurance and equity-linked insurance policies. The insurance premium is assumed to be paid as a lump-sum at $t=0$. The price process of an individual policy is given by $(9)$ as

$$
V_{H_{i}}(t)=E_{t}\left(\frac{S^{*}(t)}{S^{*}\left(T_{i} \wedge T\right)} H_{i}\right), \quad t \in[0, T]
$$

Note, to simplify notations, we assume that if $T_{i}<T$, then during the period $\left[T_{i}, T\right], V_{H_{i}}$ accumulates as the GP does over this period, so that $V_{H_{i}}$ is defined over $[0, T]$ even for $T_{i}<T$. The price process of the insurance portfolio is given by

$$
V_{\boldsymbol{H}}(t)=\sum_{i=1}^{L} V_{H_{i}}(t)=S^{*}(t) \sum_{i=1}^{L} E_{t}\left(\frac{H_{i}}{S^{*}\left(T_{i} \wedge T\right)}\right), \quad t \in[0, T] .
$$

For example, we consider a standard endowment insurance policy with a deterministic benefit of one unit of the locally risk-free savings account, payable upon the policyholder's death on or before the maturity $T$, or at $T$ if the policyholder survives. The terminal value of the $i$-th policy's fair price process is given by

$$
V_{H_{i}}(T)=\frac{S^{*}(T)}{S^{*}\left(T_{i} \wedge T\right)}
$$

The insurance portfolio's terminal value is given by

$$
V_{\boldsymbol{H}}(T)=\sum_{i=1}^{L} V_{H_{i}}(T)=\int_{0}^{T} \frac{S^{*}(T)}{S^{*}(\tau)} d N(\tau)+(L-N(T)),
$$

where the first term after the second equality sign represent the total death benefits accumulated until maturity, and the second term represent the total survival benefits. Following (9), 
the fair portfolio value process is given by

$$
V_{\boldsymbol{H}}(t)=E_{t}\left(\frac{S^{*}(t)}{S^{*}(T)} V_{\boldsymbol{H}}(T)\right)=\int_{0}^{t} \frac{S^{*}(t)}{S^{*}(u)} d N(u)+(L-N(t)) R(t), \quad t \in[0, T] .
$$

Here the first term after the second equality sign represents the fair value of the benefits paid so far, and the second term corresponds to the fair value of future benefits to be paid, where

$$
R(t)=\int_{t}^{T} s(t, u) \mu(u) P^{*}(t, u) d u+s(t, T) P^{*}(t, T), \quad t \in[0, T]
$$

may be seen as the current fair value of the "reserve account" for an individual policyholder who is still alive. This reserve account consists of a collection of fair zero-coupon bonds of different maturities. Note that the weights of zero-coupon bonds change over time, and this reserve account is not self-financing.

\section{A Brief Overview on Benchmarked Risk-Minimization}

In the model described in Sections 2 and 3 , insurance claims are contingent upon mortality information of policyholders, making these claims not fully hedgeable by trading in the financial market. For pricing in such incomplete market under the BA, we resort to the concept of benchmarked risk-minimization (BRM). In this section, we given a brief overview of the BRM. For a detailed presentation, we refer to Du and Platen (2016).

A dynamic trading strategy to deliver a contingent claim which is not perfectly hedgeable is, in general, not self-financing. We define under BRM a dynamic trading strategy as the $m+1$-dimensional stochastic process $\boldsymbol{\nu}=\{\boldsymbol{\nu}(t)=(\eta(t), \boldsymbol{\vartheta}(t)), t \in[0, T]\}$, where the vectorvalued predictable process $\boldsymbol{\vartheta}=\left\{\boldsymbol{\vartheta}(t)=\left(\vartheta_{1}(t), \ldots, \vartheta_{m}(t)\right)^{\top}, t \in[0, T]\right\}$ denotes the number of respective shares of the risky primary security accounts $\boldsymbol{S}$ held at time $t$. Here $\boldsymbol{\vartheta}$ represents the self-financing part of the trading strategy $\boldsymbol{\nu}$, where the remaining wealth of the self-financing portfolio is invested in the locally risk-free savings account; and $\eta=\{\eta(t), t \in[0, T]\}$ with $\eta(0)=0$ represents the adapted cumulative $\mathrm{P} \& \mathrm{~L}$ process of the strategy. Note that the $\mathrm{P} \& \mathrm{~L}$ process $\eta$ represents the non-self-financing part of the strategy $\boldsymbol{\nu}$, which appears in addition to the gains and losses of the self-financing part represented by the vector stochastic integral

$$
\int_{0}^{t} \boldsymbol{\vartheta}(u)^{\top} d \boldsymbol{S}(u), \quad t \in[0, T] .
$$

Here the predictable process $\boldsymbol{\vartheta}$ is assumed to be such that 25 is well-defined. The portfolio value or price process of the strategy $\boldsymbol{\nu}$ is given by

$$
V^{\boldsymbol{\nu}}(t)=V^{\boldsymbol{\nu}}(0)+\int_{0}^{t} \boldsymbol{\vartheta}(u)^{\top} d \boldsymbol{S}(u)+\eta(t), \quad t \in[0, T] .
$$


A trading strategy $\boldsymbol{\nu}$ is said to deliver an $\mathcal{F}_{T}$-measurable contingent claim $H_{T} \geq 0$ if $V^{\boldsymbol{\nu}}(T)=H_{T} \mathbb{P}$-almost surely. We call such a (potentially non-self-financing) trading strategy a hedging strategy delivering the contingent claim $H_{T}$. The price process $V^{\boldsymbol{\nu}}(t)$ can be regarded as the value of the current self-financing portfolio $V^{\boldsymbol{\nu}}(0)+\int_{0}^{t} \boldsymbol{\vartheta}(u)^{\top} d \boldsymbol{S}(u)$ plus the accumulated P\&L $\eta(t)$. Intuitively, the P\&L process can be thought of as the hedging error of the selffinancing strategy with respect to the contingent claim $H_{T}$, or equivalently the additional cost process of the strategy. The hedging strategy $\boldsymbol{\nu}$ is called replicating if $\eta(t)=0$ for all $t \in[0, T]$. The hedging strategy is called mean self-financing if the benchmarked P\&L process ${ }_{110} \hat{\eta}=\left\{\hat{\eta}(t)=\frac{\eta(t)}{S^{*}(t)}, t \in[0, T]\right\}$ forms a local martingale. The hedging strategy is said to have an orthogonal benchmarked P\&L process $\hat{\eta}$ if the process $\hat{\eta} \hat{\boldsymbol{S}}=\{\hat{\eta}(t) \hat{\boldsymbol{S}}(t), t \in[0, T]\}$ is a (vector) local martingale. Intuitively, $\hat{\eta}$ captures in a generalized least-squares sense the minimally fluctuating benchmarked P\&L process.

Conceptually, there may exist more than one hedging strategy delivering a given contingent claim $H_{T}$. In fact, almost any self-financing strategy $\boldsymbol{\vartheta}$ paired with an appropriate P\&L process $\eta$ may be a valid hedging strategy delivering the claim. BRM seeks to find an optimal hedging strategy $\boldsymbol{\nu}^{*}$, which is mean self-financing, has an orthogonal benchmarked $\mathrm{P} \& \mathrm{~L}$ process, and satisfies

$$
V^{\nu^{*}}(t) \leq V^{\nu}(t)
$$

for all $t \in[0, T]$ and every mean self-financing hedging strategy $\boldsymbol{\nu}$ delivering the contingent claim with an orthogonal benchmarked P\&L process. In other words, the BRM hedging strategy, or BRM strategy in short, is the least expensive mean self-financing hedging strategy delivering the contingent claim, with an orthogonal benchmarked P\&L process.

The optimality of the BRM strategy as described above is twofold: The strategy minimizes a generalized form of the hedging error, in that the benchmarked, unhedged part of the contingent claim forms a local martingale, orthogonal in a generalized sense to the benchmarked primary security accounts. Moreover, the initial cost of the BRM strategy is minimal among all such hedging strategies.

Not surprisingly, the price process $V^{\nu^{*}}$ of the BRM strategy $\boldsymbol{\nu}^{*}$, assuming the latter exists, is given by the real-world pricing formula

$$
V^{\boldsymbol{\nu}^{*}}(t)=E_{t}\left(\frac{S^{*}(t)}{S^{*}(T)} H_{T}\right) \quad t \in[0, T]
$$

see Du and Platen (2016), Corollary 5.4. For (28) to be useful, one needs the existence of the corresponding BRM strategy $\boldsymbol{\nu}^{*}$ for the contingent claim. For this purpose we introduce the notion of a regular contingent claim. A contingent claim is called regular if a welldefined BRM strategy $\boldsymbol{\nu}^{*}=\left(\eta^{*}, \boldsymbol{\vartheta}^{*}\right)$ exists for this claim. In other words, an $\mathcal{F}_{T}$-measurable contingent claim $H_{T} \geq 0$ is called regular if the benchmarked contingent claim $\hat{H}_{T}=\frac{H_{T}}{S^{*}(T)}$ 
assumes the representation

$$
\hat{H}_{T}=E_{t}\left(\hat{H}_{T}\right)+\int_{t}^{T} \boldsymbol{\vartheta}(u)^{* \top} d \hat{\boldsymbol{S}}(u)+\hat{\eta}^{*}(T)-\hat{\eta}^{*}(t) \quad t \in[0, T],
$$

for some predictable self-financing trading strategy process $\boldsymbol{\vartheta}^{*}$, and some local martingale $\hat{\eta}^{*}$ with $\hat{\eta}^{*}(0)=0$ as benchmarked P\&L process, which is orthogonal to $\hat{\boldsymbol{S}}$ in the sense that

$\hat{\eta}^{*} \hat{\boldsymbol{S}}=\left\{\hat{\eta}^{*}(t) \hat{\boldsymbol{S}}(t), t \in[0, T]\right\}$ forms a vector local martingale.

It should be noted that BRM makes the same minimal assumptions on the underlying financial market model as the BA. The only requirement is the existence of the GP, where all nonnegative, self-financing portfolio processes form local martingales when denominated in units of the GP. The concept of BRM is in the same spirit as classical risk-minimization as developed by Föllmer and Sondermann (1986); Schweizer (1994); Møller (1998). However, BRM allows to deliver the contingent claim with minimal cost, with less regularity conditions imposed on the market model.

\section{BRM Hedging Strategies for Endowment Insurance Policies}

Classical actuarial theory considers the risk associated with standard life insurance policies as diversifiable under the assumption that individual mortalities are independent, and that the policy benefits are not subject to common factors such as market risk. Under the BA, the latter assumption does not hold even for standard life insurance policies. In fact, benefits of the standard policies, when priced under the BA, are subject to market risk associated with the GP, c.f. (10). Fortunately, this "systematic" risk associated with the GP can be hedged by following the BRM hedging strategy, leaving only the independent mortality risk which, in principle, can be diversified away.

As an illustrative example, we consider the pricing and hedging of a homogeneous portfolio of standard endowment insurance policies as discussed in Section 2, using the BRM strategy. We assume the insurance premiums to be paid upfront at the policies' inception at $t=0$. Results obtained herein are easily modified to cover cases of different types of policies and/or premium payment schemes.

Following (28), the price process $V^{\nu^{*}}$ of the BRM strategy for the life insurance policies is the same as the one for the fair price process $V_{\boldsymbol{H}}$ given by (23), with benchmarked price process

$$
\hat{V}^{\nu^{*}}(t)=\int_{0}^{t} \frac{d N(u)}{S^{*}(u)}+(L-N(t)) \hat{R}(t), \quad t \in[0, T],
$$

and the benchmarked individual reserve account value

$$
\hat{R}(t)=\int_{t}^{T} s(t, u) \mu(u) \hat{P}^{*}(t, u) d u+s(t, T) \hat{P}^{*}(t, T), \quad t \in[0, T] .
$$


To obtain the BRM representation (28), we apply Ito's formula to (30) and exploit the fact that $\hat{V}^{\nu^{*}}$ forms a martingale. This leads to the following representation for the benchmarked price process of the BRM strategy:

$$
\hat{V}^{\boldsymbol{\nu}^{*}}(t)=\hat{V}^{\boldsymbol{\vartheta}^{*}}(t)+\hat{\eta}^{\boldsymbol{\nu}^{*}}(t), \quad t \in[0, T]
$$

with the self-financing part

$$
\hat{V}^{\vartheta^{*}}(t)=\hat{V}^{\nu^{*}}(0)+\int_{0}^{t} \gamma^{\vartheta^{*}}(\tau) \sqrt{\frac{\alpha(\tau)}{S^{*}(\tau)}} d W^{*}(\tau)
$$

where

$$
\gamma^{\vartheta^{*}}(t)=(L-N(t)) S^{*}(t) \frac{\partial \hat{R}(t)}{\partial S^{*}(t)} .
$$

The benchmarked $\mathrm{P} \& \mathrm{~L}$ process is given by

$$
\hat{\eta}^{\nu^{*}}(t)=\int_{0}^{t}\left(S^{*}(\tau)^{-1}-\hat{R}(\tau)\right) d M(\tau)
$$

The latter forms a local martingale, orthogonal to the benchmarked primary security accounts, since the mortality counting process $N$ and its compensated process $M$ are independent of the GP. To verify whether $(32)$ is in the correct form of a BRM representation, we construct the self-financing strategy $\boldsymbol{\vartheta}^{*}$ that replicates the hedgeable part $V^{\boldsymbol{\vartheta}^{*}}(t)$ of the BRM price process. By applying Ito's formula to $V^{\vartheta^{*}}(t)=\hat{V}^{\vartheta^{*}}(t) S^{*}(t)$ and considering (7), we obtain

$$
V^{\boldsymbol{\vartheta}^{*}}(t)=V^{\boldsymbol{\nu}^{*}}(0)+\int_{0}^{t}\left(\gamma^{\boldsymbol{\vartheta}^{*}}(\tau)+\hat{V}^{\boldsymbol{\vartheta}^{*}}(\tau)\right) d S^{*}(\tau)
$$

for $t \in[0, T]$. Evidently, the self-financing part of the strategy $\boldsymbol{\vartheta}^{*}$ holds the number of

$$
\delta^{\boldsymbol{\vartheta}^{*}}(t)=\gamma^{\boldsymbol{\vartheta}^{*}}(t)+\hat{V}^{\boldsymbol{\vartheta}^{*}}(t)
$$

units of the GP, and invest the remaining wealth of the self-financing part of the strategy in the locally risk-free savings account. The BRM strategy $\boldsymbol{\nu}$ for the life insurance policies is, thus, completely characterized by (30) through (37).

To gain more insights, notice that both the self-financing part of the strategy $\boldsymbol{\vartheta}^{*}$ and the P\&L process $\eta^{\nu^{*}}$ invest in the GP. In particular, the P\&L process invests at $t \in[0, T]$ a number of $\hat{\eta}^{\nu^{*}}(t)$ units in the GP. The total number of GP units held at $t \in[0, T]$ is, thus, given by

$$
\delta^{\boldsymbol{\nu}^{*}}(t)=\delta^{\boldsymbol{\vartheta}^{*}}(t)+\hat{\eta}^{\boldsymbol{\nu}^{*}}(t)=\hat{V}^{\boldsymbol{\nu}^{*}}(t)+\gamma^{\boldsymbol{\vartheta}^{*}}(t)=\int_{0}^{t} \frac{d N(u)}{S^{*}(u)}+(L-N(t)) \frac{\partial R(t)}{\partial S^{*}(t)},
$$


where the identity $S^{*}(t) \frac{\partial \hat{R}(t)}{\partial S^{*}(t)}=\frac{\partial R(t)}{\partial S^{*}(t)}-\hat{R}(t)$ is used. It is seen from 23 and 38 that $\delta^{\nu^{*}}(t)=\frac{\partial V^{\nu^{*}}(t)}{\partial S^{*}(t)}$. That is, 38 corresponds to the hedge ratio, or the "delta" of the BRM price process with respect to the GP. Evidently, the BRM strategy, effectively, resembles a deltahedging strategy, with the delta given by the sensitivity of the fair price process with respect to the GP. However, an important difference between the BRM strategy and the corresponding delta-hedging strategy is that the BRM strategy is, in general, not self-financing. It is mean self-financing in terms of the benchmarked price process under the real-world probability measure. Moreover, the real-world pricing formula (9), generally, differs from the classical risk-neutral pricing formula, yielding prices and deltas different from classical theory. We refer to the delta-hedging strategy $\delta^{\boldsymbol{\nu}^{*}}$ that effectively mimics the BRM strategy $\boldsymbol{\nu}^{*}$ as the delta-hedging implementation of the BRM strategy $\boldsymbol{\nu}^{*}$. The delta-hedging implementation of the BRM strategy differs from the exact BRM strategy only in that the exact BRM strategy compensates for any hedging errors of the delta-hedging implementation by injecting and extracting additional funds into and from the locally risk-free savings account, so that the exact BRM price, as given by (23), is maintained throughout the entire hedging process. The injection and extraction of additional funds reveal the non-self-financing nature of the BRM strategy. On the other hand, we assume that the delta-hedging implementation is self-financing. That is, while maintaining exactly the same positions in the GP, including those of the P\&L process, the delta-hedging implementation does NOT inject into or extract from the locally risk-free savings account additional funds to maintain the BRM price process 23). However, it should be noted that from a regulatory perspective, such injection and/or extraction of funds may be formally required in order to maintain the overview on the reserve account balance at or above a requested level.

\section{Numerical Examples}

To illustrate the methodology presented, we conduct some numerical experiments. In particular, we consider the pricing of a portfolio of homogeneous endowment insurance policies and the associated BRM strategy, based on the, so called, minimal market model (MMM), see Platen and Heath (2006), for the underlying discounted GP, estimated from the discounted historical prices of the S\&P500 total return index. For the mortality modelling, we take the historical mortality table for the US and estimate the historical force of mortality. We conduct Monte-Carlo simulations to assess the distribution of the P\&Ls from the BRM strategy. We further backtest the strategies on historical data and generate the P\&L process corresponding to the historically realized scenario.

When we apply the MMM, we make two important assumptions. First, the GP of a given investment universe is, generally, difficult to construct. However, it is shown by Platen and Rendek (2012) that the GP can be approximated by a well-diversified equity index. 
The MMM assumes that the S\&P500 index is a good proxy for the GP of the US domestic stock market. Second, the drift coefficient $\alpha(t)$ in the general GP model (7) is theoretically a complicated process depending on the instantaneous market prices of risks, and is, thus, difficult to specify. The MMM makes a critical simplification by assuming that $\alpha(t)$ is a simple deterministic exponential function $\alpha(t)=\alpha_{0} e^{\rho t}$. As a result, under the MMM, the GP follows a time-transformed squared Bessel process of dimension four with a deterministic time transformation, satisfying the SDE

$$
d S^{*}(t)=\left(\alpha_{0} e^{\rho t}\right) d t+\sqrt{\alpha_{0} e^{\rho t} S^{*}(t)} d W^{*}(t), \quad t \in[0, T]
$$

under the real-world probability measure $\mathbb{P}$; see Revuz and Yor $(1999)$. Here $\rho$ is the long-term growth rate of the market, and $\alpha_{0}$ is a constant representing the initial scale of the index.

We define in the model the normalized GP as $Y(t)=\frac{\rho}{\alpha_{0} e^{\rho t}} S^{*}(t), t \in[0, T]$, which satisfies the SDE

$$
d Y(t)=(1-Y(t)) \rho d t+\sqrt{Y(t) \rho} d W^{*}(t), \quad t \in[0, T] .
$$

The normalized GP is seen to be mean-reverting around the level 1. The mean-reversion of the normalized index implies a "trend reversion" of the GP around its long-term exponential growth. It is well documented that the S\&P 500 index moves in the long-run in a trend reverting pattern, where the trend is usually interpreted as a slowly moving "fundamental value" process; see Shiller (2015). By decomposing the GP into the normalized GP index $Y(t)$ and a simple exponential fundamental value function $\frac{\alpha_{0} e^{\rho t}}{\rho}$, the MMM parsimoniously captures this important stylized fact. Furthermore, the instantaneous squared volatility of the normalized GP equals that of the GP and is inversely proportional to the value of the normalized GP, generating the so-called leverage effect.

The normalized GP described by (40) is a time transformed square-root process of dimension four, with the transition law of a noncentral Chi-squared $\left(\mathrm{NCX}^{2}\right)$ distribution, given by

$$
Y(u) \stackrel{\mathcal{L}}{=} \frac{1-e^{-\rho(u-t)}}{4} \chi_{4}^{2}\left(\frac{4 e^{-\rho(u-t)}}{1-e^{-\rho(u-t)}} Y(t)\right), \quad 0 \leq t<u \leq T,
$$

where $\chi_{4}^{2}(\zeta)$ denotes a $\mathrm{NCX}^{2}$ random variable of dimension 4 and noncentrality parameter $\zeta$; see, e.g., Broadie and Kaya (2006). The $\chi_{4}^{2}(\zeta)$ random variable has finite moments of all positive orders. The probability density function $(\mathrm{PDF})$ is given by

$$
f(\zeta, x)=\frac{1}{2} e^{-\frac{\zeta+x}{2}}\left(\sqrt{\frac{x}{\zeta}}\right) I_{1}(\sqrt{\zeta x}), \quad x>0,
$$

where $I_{1}(\cdot)$ is the first order modified Bessel function of the first kind, see, e.g., Revuz and 
Yor (1999). The transition density function of the normalized index is, thus, given by

$$
p(t, Y(t) ; u, Y(u))=\frac{4}{1-e^{-\rho(u-t)}} f\left(\frac{4 e^{-\rho(u-t)}}{1-e^{-\rho(u-t)}} Y(t), \frac{4}{1-e^{-\rho(u-t)}} Y(u)\right) .
$$

It is worth mentioning that the normalized GP is dimensionless, and serves as a nontrivial

where the function $Q_{0}(a)$, given by

$$
Q_{0}(a)=\int_{0}^{\infty} a I_{1}(a x) e^{-\frac{x^{2}+a^{2}}{2}} d x, \quad a>0
$$

is a variant of the Marcum $\mathrm{Q}$ function; see Marcum (1960). From (11), at $t \in[0, T]$, the replication portfolio of the fair zero-coupon bond invests

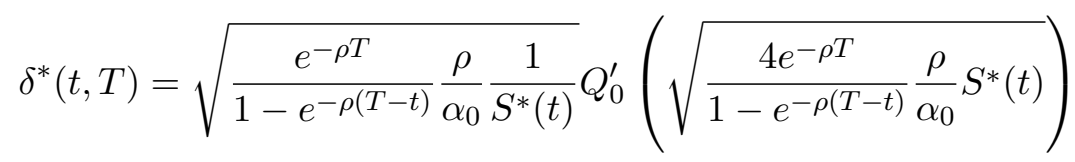

units in the GP, and its remaining value in the locally risk-free savings account.

Having introduced the MMM, we now turn to its estimation. We take as GP proxy the historically observed monthly prices of the S\&P500 index from 1871 to 2018, with all dividends reinvested, and discounted by the locally risk-free savings account. The S\&P500 data after 1963 are obtained from Datastream, and the earlier part from 1871 until 1963 is reconstructed in Shiller (2015). The S\&P500 total return index is a good approximation of the market portfolio (MP) of the US domestic stock market. As mentioned earlier, taking the MP as a GP proxy can be justified by observing that via diversification one approximates the GP of a given stock market; see Platen and Rendek (2012).

The log-prices of the S\&P500 total return index are shown in Figure 1 (a). We take the first 116 years of the available data, from Jan. 1871 to Jan. 1987, for the purpose of fitting the 
MMM, and run backtests over the remaining part of data. To estimate the overall exponential growth $\frac{S^{*}(t)}{Y(t)}=\frac{\alpha_{0}}{\rho} e^{\rho t}$ for the MMM, a straight line is fitted to the log-index prices and the slope is taken as the estimated long-term growth rate $\rho$. The scaling factor $\alpha_{0}$ is determined from the intercept. This leads to the estimates $\alpha_{0}=2.8567$ and $\rho=0.0432$. The normalized index $Y(t)$ obtained is shown in Figure 1 (b).

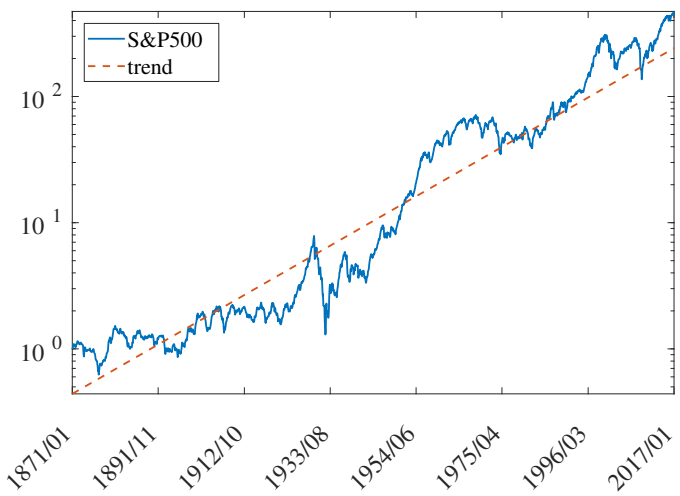

(a) S\&P500 index with estimated trend

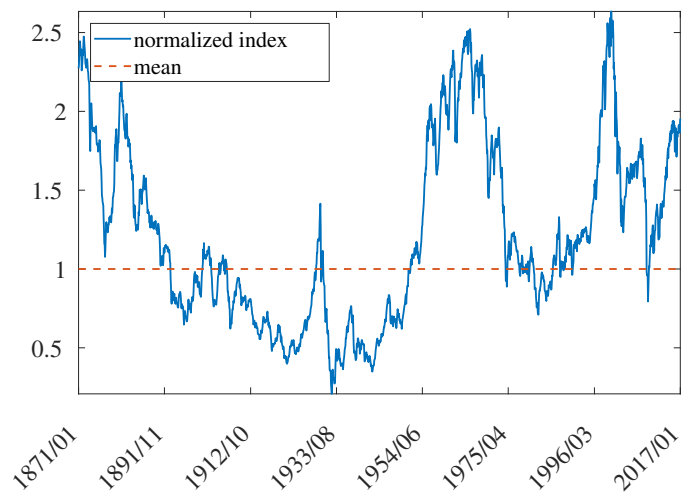

(b) Normalized index with mean

Figure 1: Estimated trend of growth and normalized index for the MMM.

To model the portfolio of life insurance policies, we assume the group of policyholders to belong to the cohort of US females aged $x=30$ at the beginning of 1987 . The portfolio consists of standard endowment insurance policies with a term of $T=30$ years. The mortality rates were obtained from The Human Mortality Database (Shkolnikov et al., 2018). We assume a yearly piecewise-constant force of mortality process for the group. From (15), the force of mortality is given by

$$
\mu(t)=-\log \left(1-q_{x+\lfloor t\rfloor}\right), \quad t \in[0, T],
$$

where $\lfloor t\rfloor$ is the integer part of $t$, with mortality rate $q_{x+\lfloor t\rfloor}:=1-s(\lfloor t\rfloor,\lfloor t\rfloor+1)$. The obtained deterministic force of mortality trajectory is shown in Figure 2 .

To gain some insights to the dynamics of the MMM, we first simulate from the fitted model a number of realized GP sample trajectories. We show the first 20 simulated sample trajectories in Figure 3 (a). We also simulate an equal number of sample trajectories of the counting process $N$, assuming a total number of $L=1000$ policies in the portfolio. We show the first 20 sample trajectories for $N$ in Figure 3 (b). It can be seen qualitatively that the market risk associated with the GP is dominant due to the considerable non-diversifiable uncertainty of the GP, as compared to the mortality counts for a moderately diversified portfolio of 1000 lives. As the number of life insurance policies increases, the mortality risk can be foreseen to be completely diversifiable (assuming the deterministic force of mortality model is correctly specified), leaving only the market risk to be hedged.

We next proceed to consider the BRM hedging strategy for the portfolio of insurance 


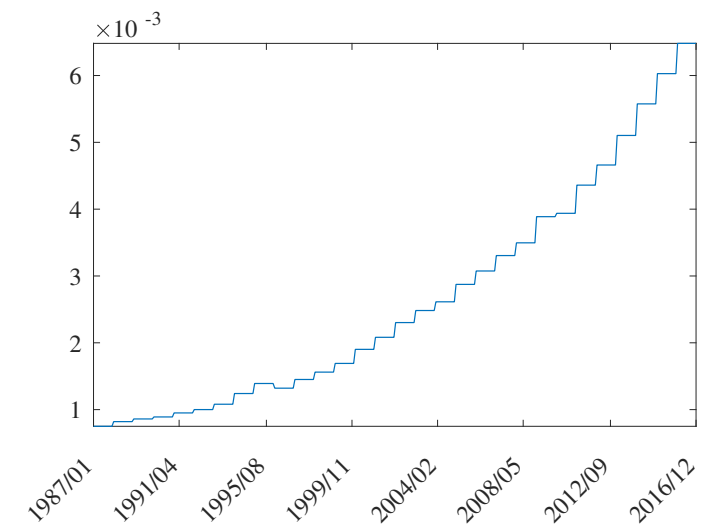

Figure 2: Force of mortality over 1987-2017 for US females aged 30 at the beginning of 1987.

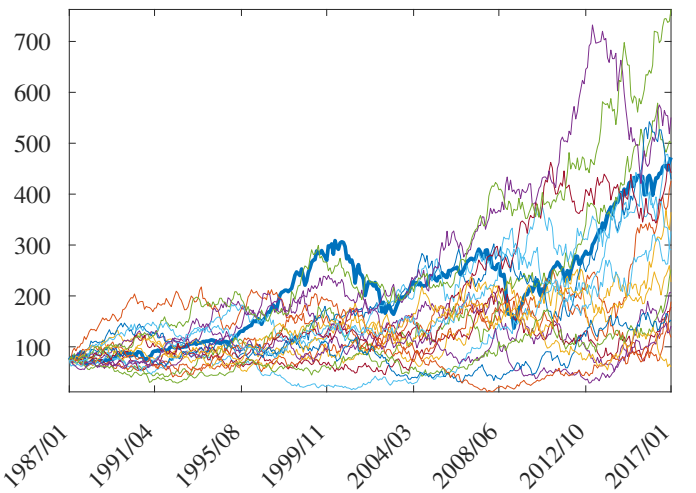

(a) GP sample trajectories

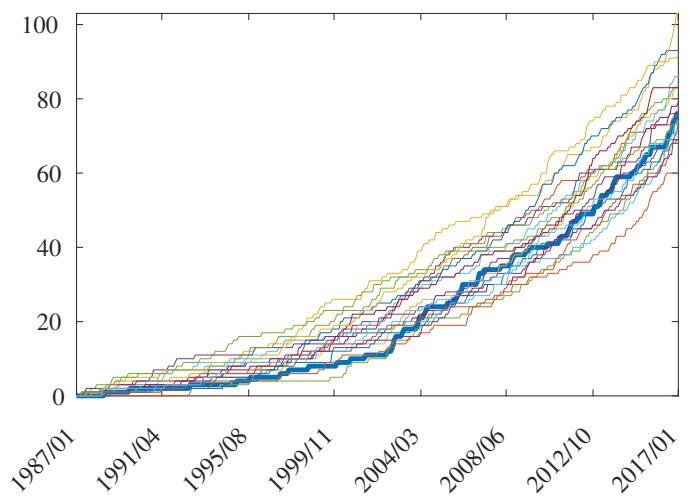

(b) Number of mortalities

Figure 3: Simulated GP sample trajectories from the estimated MMM and the number of mortalities within the portfolio from the historical force of mortality model. The thick trajectory in panel (a) is the realized S\&P 500 index prices process; The thick trajectory in panel (b) is a randomly chosen trajectory that, we assume, corresponds to the realized GP in the subsequent simulations. 
policies. We first observe from (23) that the price process of the BRM strategy for the portfolio of insurance policies consists of a combination of prices of fair zero-coupon bonds with different maturities. As a result, the BRM strategy, essentially, consists of replication strategies of fair zero-coupon bonds, given by (44) and (46) under the MMM outlined earlier in this section. It should be pointed out that the fair zero-coupon bond under the MMM is priced less expensively than under the classical risk-neutral pricing theory, where a zerocoupon bond is priced precisely at its face value. Consequently, the BRM strategy under the MMM should be priced less expensively than under the classical theory.

Following (23), we compute the BRM price trajectories along the simulated scenarios. We show for the first 20 scenarios the realized BRM price trajectories in Figure 4 (a), and in Figure 4 (b) the corresponding benchmarked price trajectories. We see that the benchmarked price trajectories seem to be trendless, while the price trajectories exhibit a visible positive trend. In fact, the sample mean of the benchmarked price trajectories stays roughly constant over time, while the sample mean of the price trajectories exhibits on average a $2 \%$ annual growth. Note that under the risk-neutral pricing theory, there should be no average net growth savings account as denominator, so that the locally risk-free interest rate is effectively zero.) By following the BA and employing the MMM, significant savings on the hedging costs for long-term insurance policies should be expected.

To assess the effectiveness of the BRM strategy, we show in Figure 4 (c) and (d) the P\&L and benchmarked P\&L trajectories, respectively. It can be seen that the benchmarked P\&L processes show very little variances for most sample trajectories. Comparing with Figure 4 (a) and (b), the P\&L trajectories evidently show much less variances than the portfolio value trajectories. The small variance in the $\mathrm{P} \& \mathrm{~L}$ trajectories indicates that the risk associated with the portfolio of insurance policies can be effectively hedged by following the BRM strategy, where the hedgeable part of the price process dominates the unhedgeable part, even with only 1000 life insurance policies.

From we compute the number of GP units held by the self-financing strategy $\delta^{\boldsymbol{\vartheta}^{*}}$ and simulate the realized price process $\tilde{V}^{\vartheta^{*}}$ by following the strategy $\boldsymbol{\vartheta}^{*}$. We show these realized price trajectories in Figure 5 (a), and the corresponding total hedging error trajectories $V^{\nu^{*}}-\tilde{V}^{\vartheta^{*}}$ in Figure 5 (c), for selected scenarios. It should be noted that the total hedging error process is given by the sum of the BRM P\&L process $\eta^{\nu^{*}}$ and the hedging error process of the self-financial part. On the other hand, the "pure" hedging errors due to imperfect, discrete hedging, as well as potential inaccuracies of the MMM, can be assessed by examining the hedging errors of the delta-hedging implementation of the BRM strategy. 265 Figure 5 (b) shows the realized delta-hedging price processes $\tilde{V}^{\delta^{*}}$, and Figure 5 (d) shows the corresponding "pure" hedging errors $V^{\nu^{*}}-\tilde{V}^{\delta^{*}}$, under the selected scenarios. It is seen from these simulations that the total hedging error of the self-financing part is dominated by the 

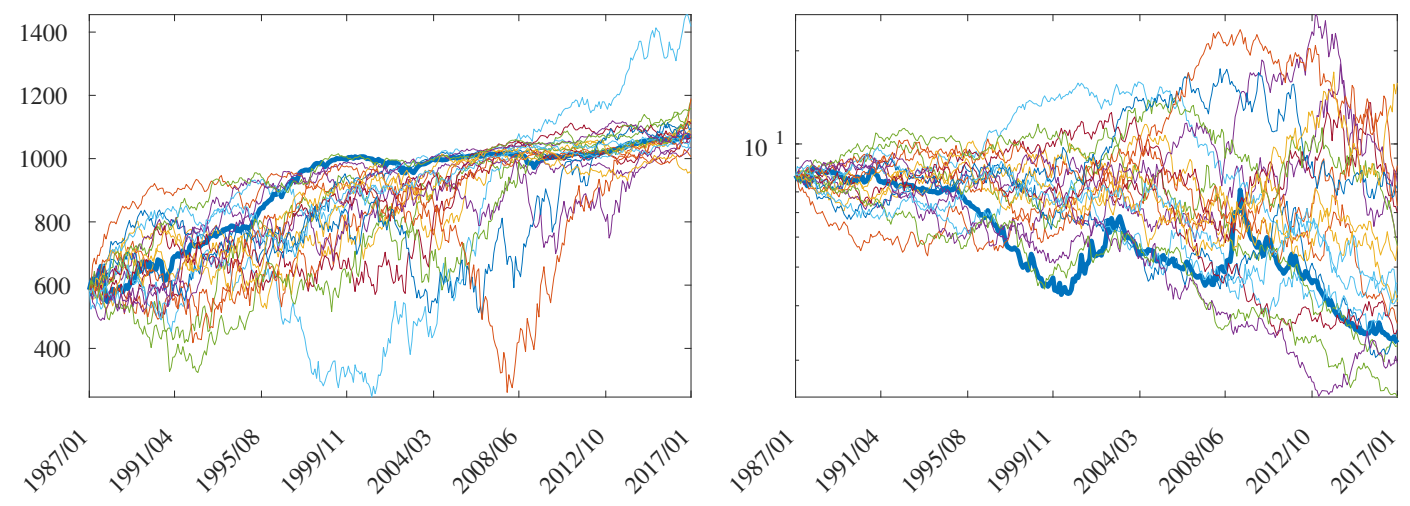

(a) BRM price processes

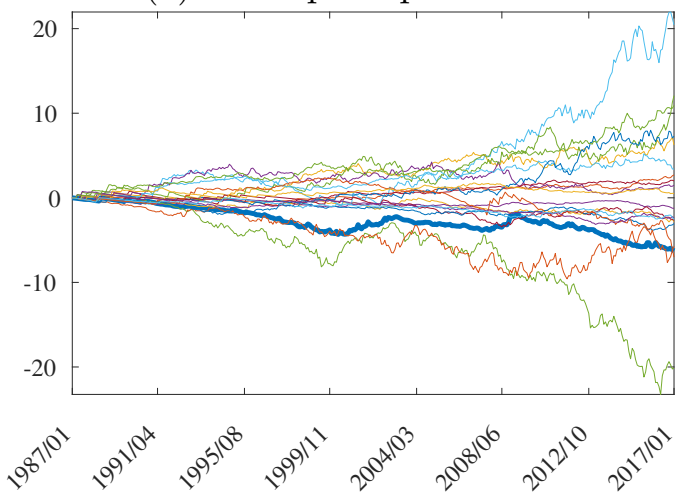

(c) P\&L processes

(b) Benchmarked BRM price processes

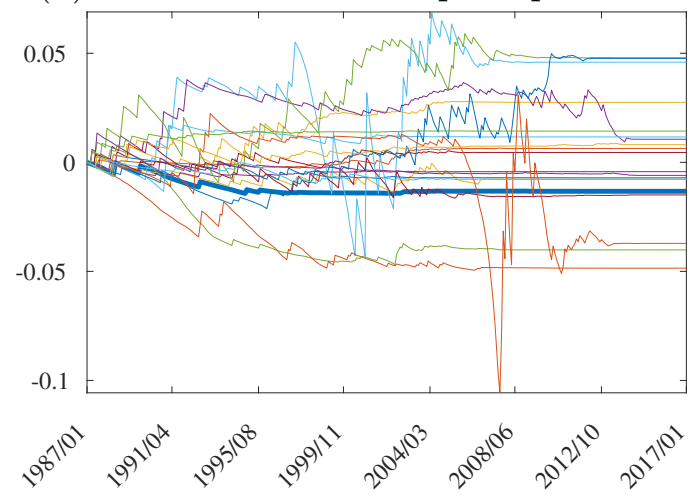

(d) Benchmarked P\&L processes

Figure 4: The sample trajectories of the BRM price process of the portfolio of life insurance policies and the benchmarked price process for the first 20 simulated scenarios, where the thick trajectories represent the realized scenario. 

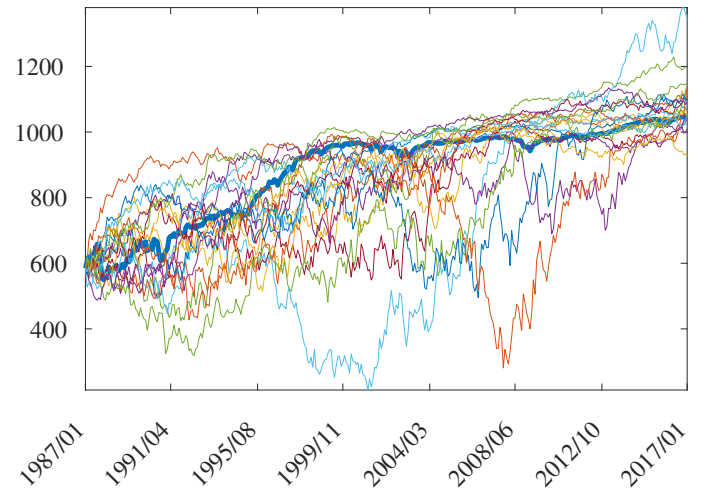

(a) Realized self-financing portfolio prices
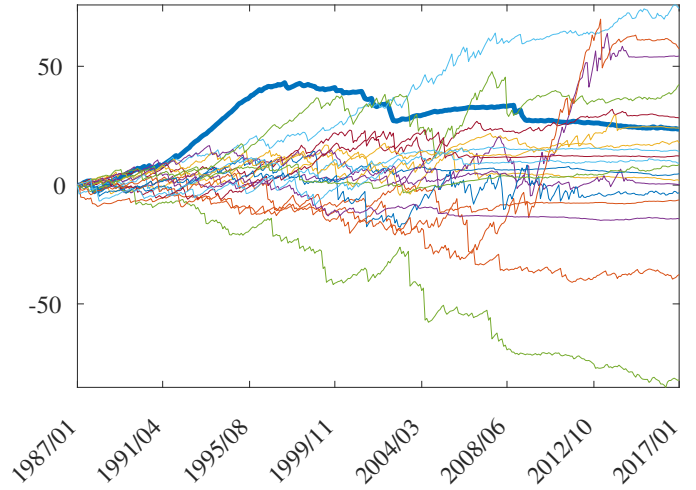
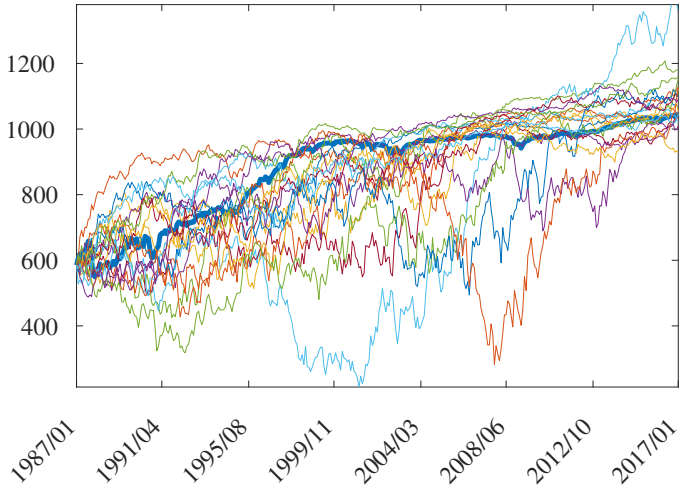

(b) Realized delta-hedging portfolio prices
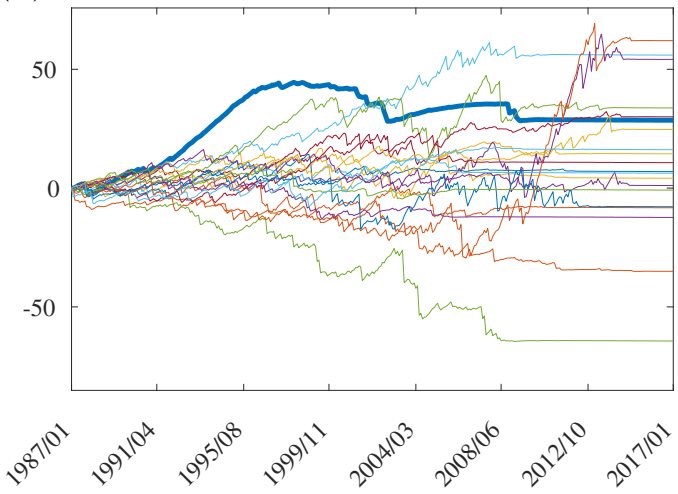

(c) Hedging errors of the self-financing portfolio (d) Hedging errors of the delta-hedging portfolio

Figure 5: The trajectories of the realized price process and the hedging errors. Panel (a) shows the realized self-financing part of the BRM price process $\tilde{V}^{\boldsymbol{\vartheta}^{*}}$; Panel (c) shows the corresponding hedging error process $V^{\nu^{*}}-\tilde{V}^{\vartheta^{*}}$; Panel (b) shows the realized delta-hedging implementation of the BRM price process $\tilde{V}^{\delta^{*}}$; and Panel (d) shows the corresponding hedging error process $V^{\boldsymbol{\nu}^{*}}-\tilde{V}^{\delta^{*}}$. 
Table 1: Sample variance reductions of terminal portfolio values, P\&Ls and hedging error processes

\begin{tabular}{r|r|r|r|r|c|c}
\hline term. val. & $\left|V^{\boldsymbol{\vartheta}^{*}}(T)\right| \eta^{\boldsymbol{\nu}^{*}}(T)$ & $\tilde{V}^{\boldsymbol{\vartheta}^{*}}(T)\left|\tilde{V}^{\delta^{\boldsymbol{\nu}^{*}}}(T)\right| V^{\boldsymbol{\nu}^{*}}(T)-\tilde{V}^{\vartheta^{*}}(T) \mid V^{\boldsymbol{\nu}^{*}}(T)-\tilde{V}^{\delta^{\nu^{*}}}(T)$ \\
\hline var. & 0.8846 & 0.0324 & 1.0435 & 1.1474 & 0.1606 & 0.1282 \\
benchmarked var. & 0.9958 & 0.0005 & 1.0299 & 1.0345 & 0.0098 & 0.0092 \\
\hline
\end{tabular}

model and discretization errors. This should not be surprising since the market risk in this case is the dominant risk factor after the mortality risk has been largely diversified.

As a final step, we show in Table 1 the sample variances of the terminal values of various price trajectories and their hedging errors, denominated both in the locally risk-free savings account and in the GP, respectively, where the variances are normalized by sample variances of the BRM price trajectories.

\section{Summary}

In this paper, we considered pricing and hedging of standard life insurance policies under the BA. Under our setting, the classical risk-neutral pricing measure may not exist, and standard life insurance policies are subject to both mortality risk and market risk. The mortality risk cannot be hedged by trading in the financial market. We employ benchmarked risk minimization (BRM) as a generalized concept of risk minimization, and developed the BRM hedging strategy for a homogeneous portfolio of standard endowment insurance policies subject to a deterministic force of mortality. We conducted numerical simulation studies under the MMM, based on historical equity market and mortality data, and demonstrated a BRM hedging strategy for a fictional portfolio of life insurance policies subject to historical market and mortality risks. Our studies revealed good hedging outcomes over simulated, as well as, historical scenarios with small hedging errors. Moreover, the BRM hedging strategy realized significant growth over the period of the insurance policies beyond that of the riskfree interest rate. This implies significant savings on the hedging costs of the insurance policies, which may be passed on to the insurance company as additional profits and/or to the policyholders as reduced premiums. The approach developed in this paper can be generalized to more complicated insurance policies, including equity-linked contracts, etc., and more realistic market and mortality models.

\section{References}

Bauer, D., Kling, A., Russ, J., 2008. A universal pricing framework for guaranteed minimum benefits in variable annuities. ASTIN Bulletin 38 (2), 621-651. 
Broadie, M., Kaya, O., 2006. Exact simulation of stochastic volatility and other affine jump diffusion processes. Operational Research 54, 217-231.

Dickson, D. C., Hardy, M., Hardy, M. R., Waters, H. R., 2013. Actuarial Mathematics for Life Contingent Risks. Cambridge University Press.

Du, K., Platen, E., 2016. Benchmarked risk minimization. Mathematical Finance 26, 617-637.

Föllmer, H., Sondermann, D., 1986. Hedging of non-redundant contingent claims. In: Hildebrandt, W., Mas-Colell, A. (Eds.), Contributions to Mathematical Economics. North Holland, pp. 205-223.

Karatzas, I., Shreve, S. E., 1991. Brownian Motion and Stochastic Calculus, 2nd Edition. Springer.

Marcum, J., 1960. A statistical theory of target detection by pulsed radar. IRE Transactions on Information Theory 6 (2), 59-267.

Møller, T., 1998. Risk-minimizing hedging strategies for unit-linked life insurance contracts. ASTIN Bulletin: The Journal of the IAA 28 (1), 17-47.

Platen, E., Heath, D., 2006. A Benchmark Approach to Quantitative Finance. Springer Finance. Springer.

Platen, E., Rendek, R., 2012. Approximating the numeraire portfolio by naive diversification. Journal of Asset Management 13 (1), 34-50.

Revuz, D., Yor, M., 1999. Continuous Martingales and Brownian Motion, 3rd Edition. Springer.

Schweizer, M., 1994. Risk minimizing hedging strategies under restricted information. Mathematical Finance 4 (4), 327-342.

Shiller, R. J., 2015. Irrational Exuberance: Revised and expanded third edition. Princeton University Press.

Shkolnikov, V., Barbieri, M., Wilmoth, J., 2018. The Human Mortality Database. 